\author{
Reto Meuli \\ Yeukuang Hwu \\ Jung Ho Je \\ Giorgio Margaritondo
}

\section{Synchrotron radiation in radiology: radiology techniques based on synchrotron sources}

Received: 18 November 2003

Revised: 14 April 2004

Accepted: 23 April 2004

Published online: 31 July 2004

(C) Springer-Verlag 2004

R. Meuli (

Service de Radiodiagnostic et Radiologie Interventionnelle,

CHUV, 1011 Lausanne, Switzerland

e-mail: reto.meuli@hospvd.ch

Tel.: +41-21-3144559

Fax: +41-213144554

\section{Y. Hwu}

Institute of Physics,

Academia Sinica,

Nankang, Taipei, 11529, Taiwan, ROC

J. H. Je

Department of Materials Sciences,

Pohang University of Science

and Technology,

Pohang, South Korea

G. Margaritondo

Faculté des Sciences de Base,

Ecole Polytechnique Fédérale de Lausanne (EPFL),

1015 Lausanne, Switzerland

\begin{abstract}
The characteristics of synchrotron X-ray sources-quite different from those of conventional sources - are exploited by several new imaging techniques. These techniques expand the capabilities of conventional radiology and find interesting application in special cases. We briefly review the basic principle, applications and limitations of the most important of them: monochromatic mammography, twowavelength digital subtraction angiography, phase-contrast/edge-enhancement imaging, diffraction-enhanced imaging and microtomography.
\end{abstract}

Keywords Synchrotron - Storage ring - Undulator - Wiggler bending magnet Phase-contrast . Diffraction-enhanced imaging . Tomography · Digital subtraction

\section{Introduction}

Part I of our review [1] explained that the properties of synchrotron X-rays are quite different from those of standard sources based on the Röntgen mechanism. We saw in particular $[2,3]$ that synchrotron sources provide highly collimated and powerful (bright) beams with linear polarization (circular polarization for special devices). Specifically, undulators and wigglers provide beams collimated both in the horizontal and in the vertical directions because of the small source size and angular spread along all directions. Bending magnet sources instead produce fan-like beams with a very small angular spread in the vertical direction; the horizontal spread depends in this case on the angular range collected by the beamline.

These advanced characteristics - and in particular the emission geometry - make it difficult to compare directly a synchrotron source with standard radiology sources, conventionally characterized by the X-ray tube voltage and by the tube current. As a rough comparison, a bending magnet synchrotron source is at least 5-6 orders of magnitude brighter than a conventional source; an advanced "wiggler" synchrotron source can beat the brightness of a conventional emitter by 12 orders of magnitude or more. 
(a)
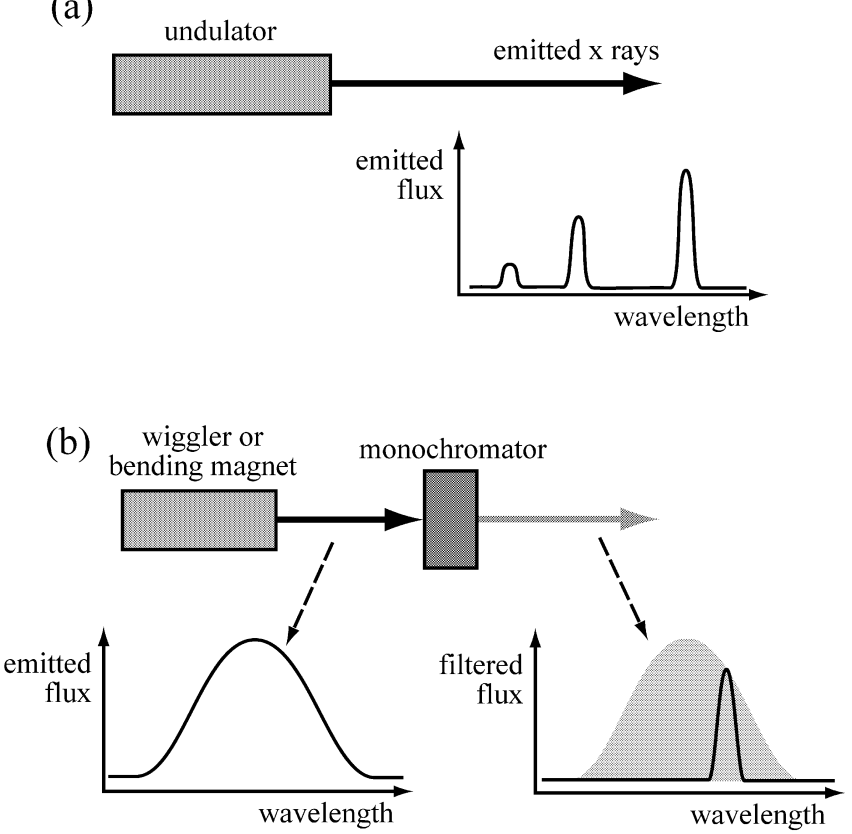

Fig. 1 Synchrotron sources can provide X-rays of well-defined wavelength (or, rather, narrow bands centered at the desired wavelength). a An undulator emits only very narrow bands of wavelengths. Individual bands can be selected and the center can be changed by changing the undulator parameters; $\mathbf{b}$ a bending magnet or a wiggler emits instead a broad band from which the desired wavelength can be filtered with a monochromator

The wavelength of synchrotron X-rays can be adjusted over a large spectral range. More specifically (Fig. 1), an undulator emits only specific wavelengths (or, rather, very specific bands of wavelengths) that can be modified by changing the working parameters of the undulator. A bending magnet or a wiggler emits a very broad band of wavelengths from which the desired value can be filtered with a device called a monochromator.

The emission of synchrotron sources is highly coherent, producing detectable diffraction and interference phenomena that can be used for imaging. Finally, the emission has a time structure consisting of short pulses separated by longer dark intervals that is used in time-resolved techniques $[2,3]$.

All such characteristics can be exploited to image objects in non-conventional ways. The corresponding techniques offer advantages and disadvantages with respect to standard radiology. They cannot replace standard radiology, but can offer complementary performances and can be quite useful for specialized applications. Furthermore, their scope extends beyond medical radiology of live human patients, reaching other radiological techniques and applications of great interest to medical and non-medical research and to other fields. Accordingly, our discussion is not strictly limited to diagnostic radiology.
The technical implementation of these novel imaging approaches is often complex. On the contrary, their conceptual basis is quite simple and can be explained in elementary terms: this is the scope of part II of our review. We will discuss, in particular, the following techniques: onewavelength mammography [4-7, and references cited in 7], two-wavelength digital subtraction angiography [8-12], phase-contrast/edge-enhancement imaging [13-22], diffraction-enhanced imaging (DEI) [23, 24] and phase-contrast microtomography [25-27]. The objective of our presentation is to provide for non-specialized readers a simple picture of each technique: we neither intend to present a detailed report on the results of each technique nor a complete list of references. Table 1 presents a summary of the characteristics and applicability of the techniques we discuss.

\section{Techniques based on wavelength selection: mammography and angiography}

The possibility to take radiological images with only one wavelength (monochromatized X-rays: see Fig. 1) in many cases has had an immediate positive impact: the diffused background of the image is reduced with respect to unmonochromatized X-rays. Keeping the X-ray dose constant, this increases the image contrast and quality.

A good example of this positive aspect is provided by mammography. The sources that are typically used for dedicated mammography systems are molybdenum anode X-ray tubes. Their wavelength spectrum consists of two strong peaks ("lines") at approximately $0.63 \AA$ and $0.71 \AA$ (corresponding to photon energies of approximately $19.4 \mathrm{keV}$ and $17.6 \mathrm{keV}$ ). These wavelengths do fall in the optimal spectral region for mammography [0.59-0.73 $\AA(17-21 \mathrm{keV})$; the actual optimum wavelength and photon energy changes with the breast thickness and density]. However, the two peaks are superimposed upon a spectral continuum due to bremsstrahlung. This continuum extends to wavelengths below the optimal range for mammography, producing a diffused background that deteriorates the image contrast.

A synchrotron source makes it possible to take mammographic images with one single wavelength peak, thereby drastically reducing the diffused background [4-7, and references cited in 7]. Furthermore, the wavelength can be tuned to match the optimal wavelength for each breast thickness and density.

Synchrotron mammography tests are typically performed with a bending magnet source. We mentioned that this source produces a fan-like beam with a narrow vertical angular spread and a broader horizontal spread. The images are taken by vertically scanning the line illuminated by the beam. The vertical collimation of the beam makes it possible to insert slits behind the breast, thereby further reducing the background. 
Table 1 Summary overview of synchrotron-based radiological techniques

\begin{tabular}{|c|c|c|c|c|c|}
\hline & $\begin{array}{l}\text { Synchrotron } \\
\text { mammography }\end{array}$ & $\begin{array}{l}\text { Two-wavelength } \\
\text { angiography } \\
\text { (dichromography) }\end{array}$ & $\begin{array}{l}\text { Phase contrast } \\
\text { imaging }\end{array}$ & $\begin{array}{l}\text { Diffraction-enhanced } \\
\text { imaging }\end{array}$ & $\begin{array}{l}\text { Synchrotron } \\
\text { microtomography }\end{array}$ \\
\hline Wavelengths & Single & Two & $\begin{array}{l}\text { Single or } \\
\text { broadband }\end{array}$ & $\begin{array}{l}\text { Single or } \\
\text { broadband }\end{array}$ & $\begin{array}{l}\text { Single or } \\
\text { broadband }\end{array}$ \\
\hline $\begin{array}{l}\text { Current } \\
\text { applications }\end{array}$ & $\begin{array}{l}\text { Phantoms, } \\
\text { anatomical } \\
\text { specimens }\end{array}$ & Live patients & $\begin{array}{l}\text { Anatomical and } \\
\text { biological } \\
\text { specimens }\end{array}$ & $\begin{array}{l}\text { Anatomical and } \\
\text { biological } \\
\text { specimens }\end{array}$ & $\begin{array}{l}\text { Anatomical and } \\
\text { biological } \\
\text { specimens }\end{array}$ \\
\hline $\begin{array}{l}\text { Potential } \\
\text { applications }\end{array}$ & Live patients & $\begin{array}{l}\text { Routine examinations } \\
\text { of live patients }\end{array}$ & Live patients & Live patients & $\begin{array}{l}\text { Possibly live } \\
\text { patients }\end{array}$ \\
\hline Problems & $\begin{array}{l}\text { Not yet a certified } \\
\text { technique for } \\
\text { medical use }\end{array}$ & $\begin{array}{l}\text { Complicated } \\
\text { implementation }\end{array}$ & $\begin{array}{l}\text { Non-conventional } \\
\text { image features, } \\
\text { require special } \\
\text { interpretation } \\
\text { techniques }\end{array}$ & $\begin{array}{l}\text { Non-conventional } \\
\text { image features, require } \\
\text { special interpretation } \\
\text { techniques }\end{array}$ & $\begin{array}{l}\text { For phase-contrast: } \\
\text { non-conventional } \\
\text { image features, } \\
\text { require special } \\
\text { interpretation } \\
\text { techniques }\end{array}$ \\
\hline $\begin{array}{l}\text { Time per } \\
\text { image }\end{array}$ & $1-10 \mathrm{~s}$ & Tens of milliseconds & $<1 \mathrm{~ms}$ & Down to milliseconds & $\begin{array}{l}\text { Several seconds } \\
\text { (for complete } \\
\text { image set) }\end{array}$ \\
\hline
\end{tabular}

The notion that synchrotron radiation can drastically reduce the diffused scattering background in mammograhy was first tested at the Frascati National Laboratory $[4,5]$ and then in other facilities $[6,7$ and references cited in 7]. These tests were quite positive: the image quality could be preserved while substantially reducing the required dose of X-rays. Conservatively, the scattered-radiation reduction is at least by a factor of 100 (and much larger in the phase-contrast geometry explained later), contributing to an overall dose reduction for comparable image quality of $20-60 \%$.

Wavelength tunability is also the essential ingredient of another emerging technique: dichromography [8-12]. This approach is used for coronary angiography during intravenous injection of contrast material.

Synchrotron dichromography (Fig. 2) is a variant of digital subtraction angiography. Rather than being taken at different times before and after iodine injection, the two images are taken simultaneously at two different wavelengths after iodine injection-for example, by illuminating the object with two different X-ray beams and detecting them with two different detectors (see later, Fig. 3a). This removes the motion effects of the heartbeat. The wavelengths fall right below and right above the main X-ray absorption edge of iodine (K-edge, at a wavelength of $0.37 \AA$ ) - see Fig. 2. The edge corresponds to a sharp increase in the iodine-related absorption; thus, the digital subtraction does enhance the iodine-related features with respect to the other features.

Practically speaking, the sensitivity to iodine can increase by two orders of magnitude, drastically reducing the required iodine concentration. The contrast agent can be inserted with a relatively safe intravenous injection procedure. The dilution by a factor $\approx 50$ during the transit 

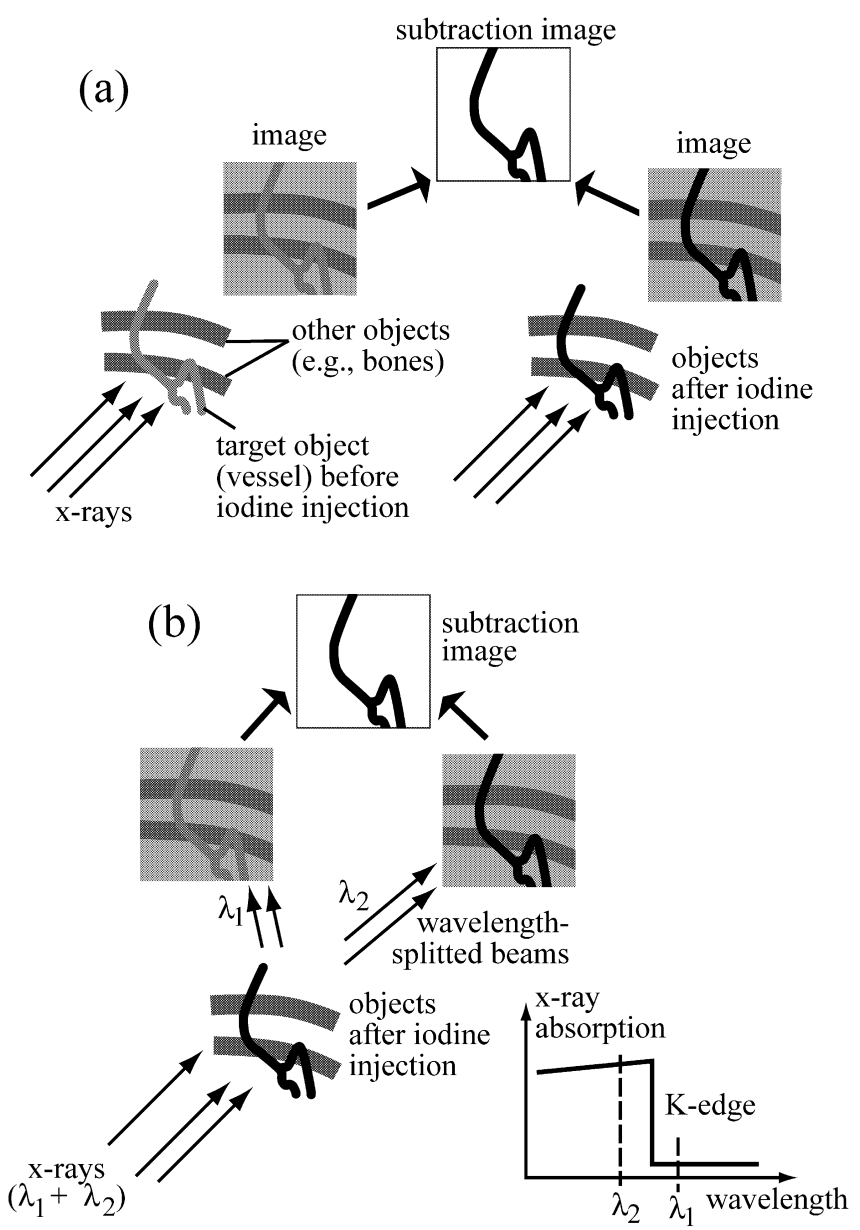

Fig. 2 a Digital subtraction angiography: images taken with and without the contrast agent (iodine) are subtracted from each other, enhancing the iodine-containing features. In the heart the procedure is affected by the motion since the two images are taken at different times. b Dichromography: images of the object with the contrast agent are taken simultaneously with two wavelengths above and below the main X-ray absorption edge of the contrast agent. The digital subtraction enhances once again the iodine-containing features and is not affected by the motion of the heart

to the coronary region is in fact compensated by the increase in sensitivity to iodine.

Several synchrotron facilities are actively engaged in experimental angiography programs based on dichromography [8-12]. The approach of centers like HASYLAB in Hamburg and Brookhaven [8, 9]schematically illustrated in Fig. $3 a-$ is based on "line scanning." A horizontal line of unmonochromatized synchrotron radiation goes through a specially designed monochromator that produces two fan-shaped beams with the two desired wavelengths. The optical system is designed so that the two beams cross at the position of the patient's heart and are separately detected afterwards. Two-dimensional images are obtained by scanning, i.e., by moving the patient vertically across the beam. Images
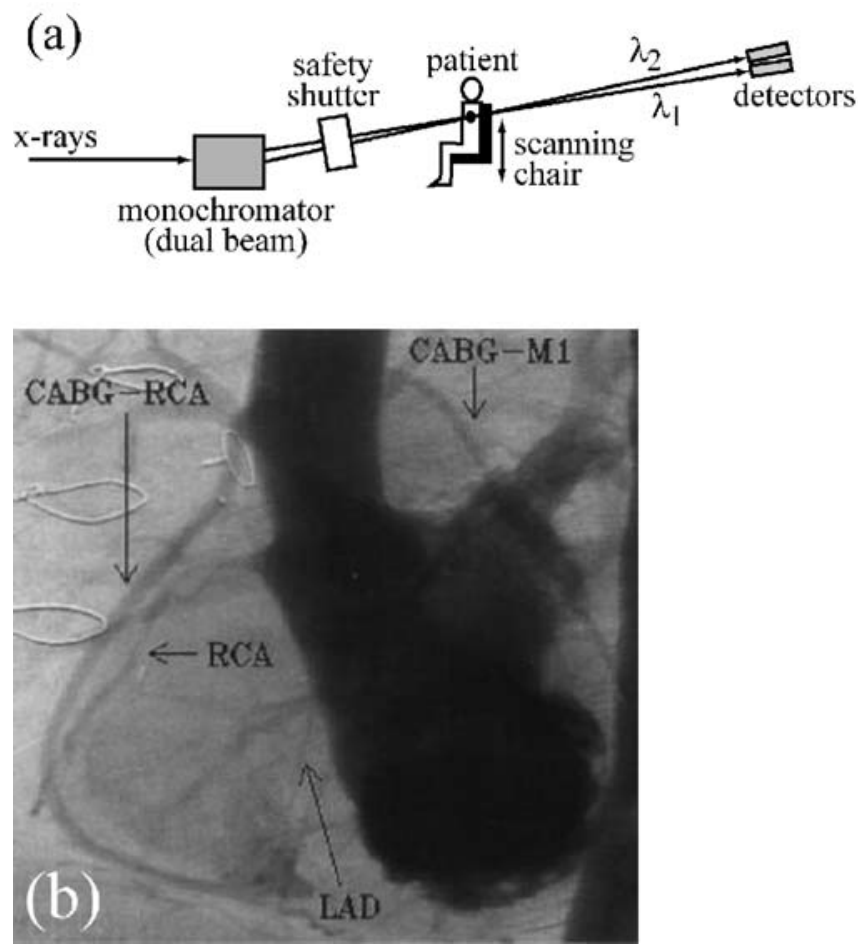

Fig. 3 a Scheme of a system for digital subtraction angiography performed with the dichromography approach (see [8, 9]). b Intravenous synchrotron angiogram of venous coronary artery bypass grafts to right coronary artery (CABG-RCA) and marginal branch (CABG-M1) in left anterior oblique projection taken with the NIKOS system at HASYLAB. (Courtesy W.R. Dix—see [8])

are acquired during diastole with a sufficient speed of the patient enabling a complete scanning of the heart in less than $250 \mathrm{~ms}$.

The dichromography system of the European Synchrotron Radiation Facility (ESRF) in Grenoble [11] is an excellent practical example of this approach. The patient chair moves with a speed of $250 \mathrm{~mm} / \mathrm{s}$ and the frame rate is $1.4 \mathrm{~ms}$, corresponding to a resolution of $350 \mu \mathrm{m}$. The typical X-ray dose is $30 \mathrm{mGy}$ per image. In case of failure, emergency systems can shut down the $\mathrm{X}$-ray beams in $10 \mathrm{~ms}$ to avoid an excessive dose.

Figure $3 \mathrm{~b}$ shows one example of the many tests already performed on live patients with this approach. Note that the line-scan technique is automatically insensitive to the scattered flux, eliminating the need for an anti-scatter grid. The line detector was in this case a 13-cm-wide ionization chamber filled with $\mathrm{KrCO}_{2}$.

Two-dimensional systems for dichromography were developed in Japan that do not require the scanning procedure. The strategy used at the TRISTAN source [12] is based on a non-conventional elliptical wiggler that produces two slightly diverging beams. As the two beams pass through a specially designed monochromator, they are spread to horizontal and vertical sizes of several cen- 
timeters, suitable for a two-dimensional angiographic image. The monochromator provides spectral filtering so that the two final beams have the two desired wavelengths. In addition, scientists in Japan have extensively used monochromatic synchrotron radiation for angiography without digital subtraction [12].

Extensive tests were performed on synchrotron dichromography of human patients, notably at HASYLAB in Hamburg [8]. The future impact of this technique is difficult to assess in light of the general complication in using synchrotrons for radiological analysis, discussed in Part I and in the final chapter. There may also exist the possibility to replace iodine with other contrast agents such as gadolinium, whose edge occurs at a wavelength of $0.31 \AA$, shorter than the iodine edge of $0.37 \AA$ : tests on this approach are underway.

\section{Techniques based on collimation and coherence: refractive-index imaging}

We saw [1] that the emission of a synchrotron source is spread over a very narrow range of angles in the vertical direction; the angular collimation is equally strong in the horizontal direction for wigglers and undulators. Furthermore, the source size is very small. A source with strong angular collimation and small size is said to be "spatially coherent."

Coherent sources of visible light such as the lasers produce diffraction and interference phenomena. The same is true for coherent X-rays. Diffraction and interference can lead to image contrast with non-conventional and rather effective mechanisms $[7$ and references cited in 7, 13-22], often called (somewhat inaccurately) "phase contrast."

The coherence-based contrast mechanisms are related to the "refractive index"- the parameter characterizing the phenomena of refraction, interference and diffraction. Such phenomena are negligible in standard radiology, whose contrast is due to the different X-ray absorption by different parts of the object.

Absorption is in fact an important mechanism in the interaction between the X-rays and the object. However, other mechanisms are also active. Scattering phenomena produce a diffused background that decreases the image quality. Refraction, interference and diffraction can also occur, but their impact on standard radiology is rather limited.

Figure 4, for example, shows the direct comparison of two radiographs of the same objects taken with different experimental conditions that reduce or enhance these non-conventional mechanisms. The bottom image is quite striking, although the X-ray dose was lower than for the top image. Note that the "quality" of the bottom image is due to the enhanced visibility of the edges between different regions.
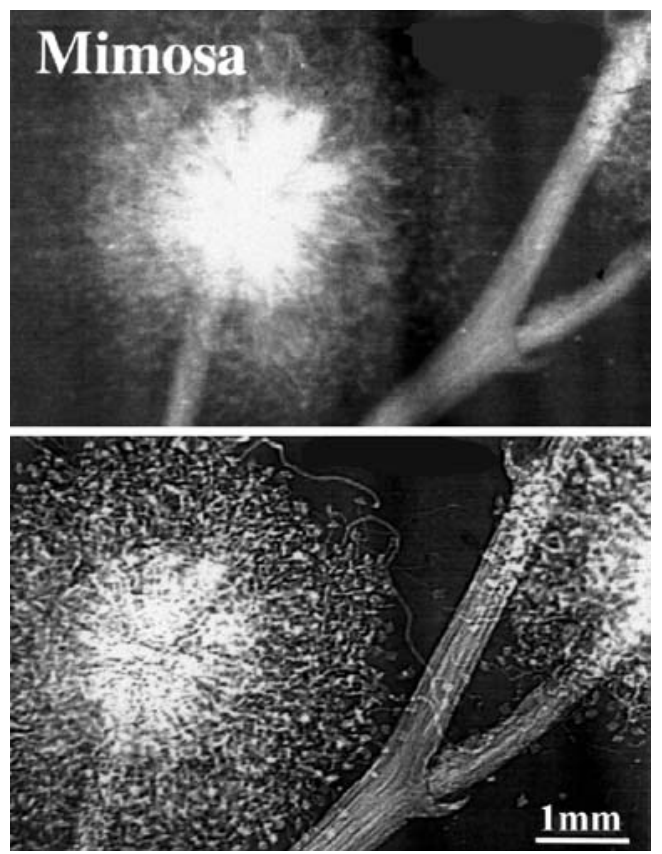

Fig. 4 Direct comparison of two radiographs of the same mimosa flower taken with the absorption contrast of standard radiology (top) and with "phase contrast" (bottom). Image obtained by Arfelli et al. at the ELETTRA lanboratory in Trieste (see $[7,14,15]$ )

The leading contrast mechanism in Fig. 5 is related to refraction and is schematically illustrated in Fig. 5. Consider the case of Fig. 5a in which the X-ray beam passes trough the tapered edge separating two different regions of the object. For the sake of simplification, assume that the two regions have similar X-ray absorption but (slightly) different X-ray refraction. As shown in Fig. 5a, the corresponding beam deviation by the edge region produces a bright-dark fringe pair on the detector-and enhances the visibility of the edge. This is precisely what is observed in the bottom image of Fig. 4. The mechanism is somewhat similar to the interaction of visible light with the edge of a glass that makes it more visible than the glass itself.

Figure $5 \mathrm{~b}$ indicates that a small source size, one of the characteristics of a coherent source, is required to see the refraction-related edge enhancement. If the source is too large, penumbra-like effects wash out the edge enhancement. A conventional X-ray source could in principle be converted into a small-size source by using a screen with a pinhole. The side effect, however, is to waste most of the source emission. A synchrotron source is instead naturally small (at least in the vertical direction).

The actual geometric conditions for refraction-based edge enhancement are not very stringent [22]: virtually all synchrotron sources built after the mid-1980s are sufficiently small. Furthermore, a "monochromatic" (singlewavelength) beam is not required [22]. This eliminates 
(a)
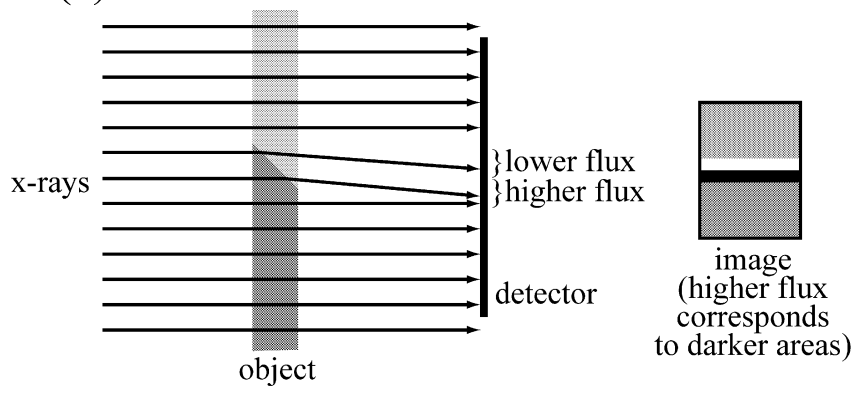

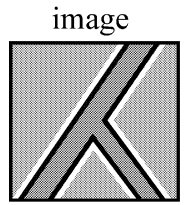

(b)

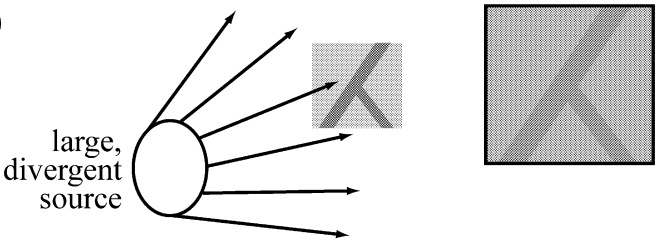

Fig. 5 a Schematic explanation of the mechanism of edge enhancement due to refraction in the X-ray image of the tapered edge between two regions of the object. b A small and collimated source certainly gives the edge enhancement; for a large and divergent source, the enhancement may be washed out by penumbra effects

the need for monochromator filtering and the consequent loss of X-ray flux.

Before presenting some example of edge-enhanced radiographs, we would like to compare refraction-based contrast to the standard absorption contrast of conventional radiology. Absorption by soft biological materials is quite low for the relevant wavelengths: this is what enables radiology to "see" inside objects. For example, absorption attenuates an X-ray beam with $30 \mathrm{keV}$ photon energy to one-half of the original intensity within $\approx 19 \mathrm{~mm}$ in soft tissues $(\approx 4.3 \mathrm{~mm}$ in bones). Small absorption corresponds to small absorption differences between soft tissues. In some cases, the differences are so small that they create image quality problems, for example, in mammography.

Refraction is also very small for X-rays and so are the differences between materials. The refraction-induced beam deviations are determined by the difference between the refractive index of the material and unity (the refraction index of vacuum). This difference is very small for all materials. For example, the refractive index

of water (soft tissues) for $30 \mathrm{keV}$ photon energy deviates from that of vacuum only by one part in 10 billion.

In spite of this, however, refraction can be more effective than absorption in producing image contrast. This is due to two factors. First, X-ray refraction effects and their differences between different materials, although small, are comparatively more pronounced than absorption effects. Second, refraction contrast is a geometrically localized effect: even small X-ray deviations are very effective in producing fringes and in increasing the edge visibility - as seen in the bottom image of Fig. 4. Therefore, edge-enhanced radiography could advantageously complement standard radiographs when low-absorption contrast creates problems.

\section{Examples of images with refraction-induced index edge enhancement}

The practical use of edge-enhancement contrast requires meeting certain experimental conditions [21, 22]. The detectors must have sufficient lateral resolution to reveal the pair of narrow dark-bright fringes. Figure 5a suggests that the fringe width on the detector increases with the object-detector distance. If this distance is too small, the detector resolution (a few microns) is not sufficient to reveal the fringes, and the edge enhancement is washed out. Absorption contrast dominates in this case: this is in fact how the edge enhancement was suppressed in the top image of Fig. 4.

Figure 5a also indicates that when the detector-object distance becomes too big, the refraction-based fringe pattern spreads and may no longer produce edge enhancement. In this case, edge enhancement can still occur because of the "diffraction" mechanism discussed later [21].

When all the required experimental conditions are met, refraction-induced edge enhancement can produce rather spectacular results. With an unmonochromatized ("white") beam, the signal becomes very high and highquality; detailed images can be obtained on a microscopic scale (Fig. 6a). The lateral resolution reaches submicron levels suitable for imaging individual cells and is being further improved.

White beams also make it possible to take real-time images with a time resolution that reaches the millisecond level and is also rapidly improving. This recently led to the first successful tests of edge-enhanced radiology on live specimens $[13,28]$. Figure $6 \mathrm{~b}$ shows a typical example of such results: microscopic structures of a live millimeter-size fish.

Time and lateral resolution play a very important role in non-medical applications of radiology [13]. Microradiology movies, for example, can solve long-standing issues in material sciences phenomena by direct real-time observation. Figure 7 provides an excellent example: the direct 

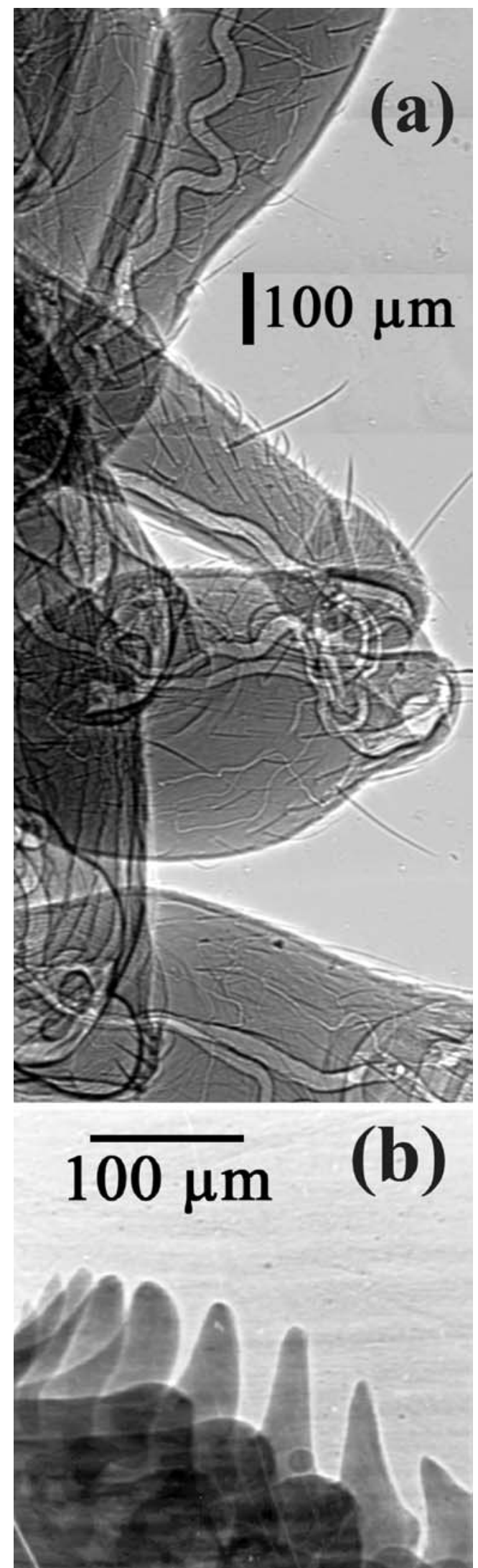

Fig. 6 Examples of images with refraction-induced edge enhancement [13]. a Microscopic features of an ant leg are sharply visible in this edge-enhanced white-beam image. b One of the first tests on live organisms: bone microstructure of a live millimeter-size fish

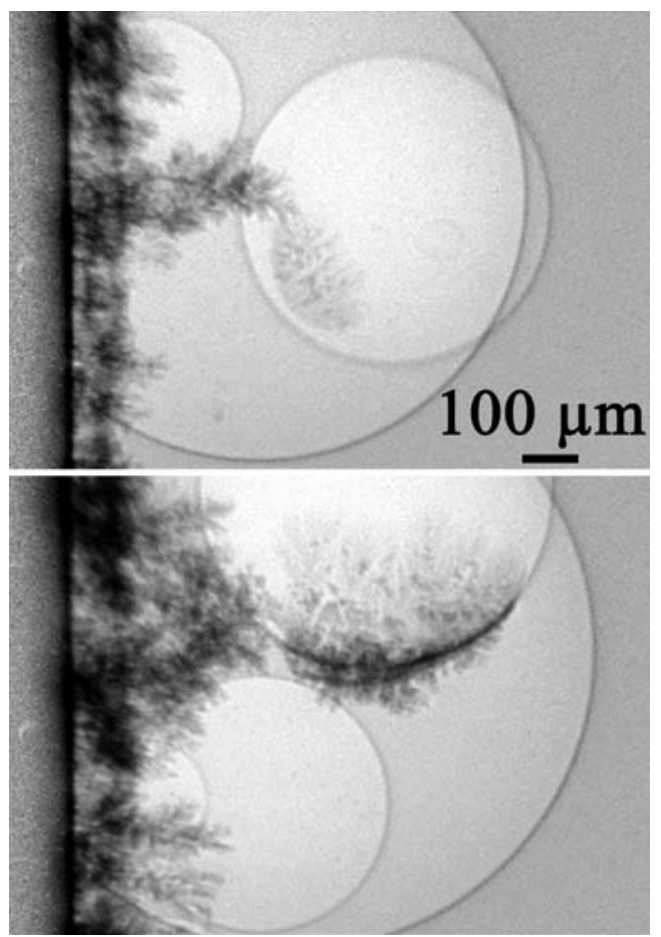

Fig. 7 Real-time edge-enhanced microradiology applied to material science. The two frames captured from a radiology movie [28] reveal a very surprising phenomenon during a metal coating procedure: the metal grows on gas bubbles forming on the substrate during the deposition process

detection of an almost incredible phenomenon in the electrodeposition of thin metal coatings [29]. As clearly seen in the radiology movie frames, the electrodeposition process creates hydrogen bubbles at the substrate-coating interface. The metal coating grows on the fast-developing bubbles; as these evaporate, spherical voids are left that finally explain a common kind of coating defects.

\section{Diffraction phenomena in edge enhancement}

Our treatment of edge enhancement was so far limited to simple refraction phenomena [22]. Reality is a bit more complicated. First of all, the edges between different object regions do not typically have simple profiles like that of Fig. 5a. This is a source of complications, but also, potentially, of additional information.

Our analysis was also oversimplified from the point of view of fundamental optics. A complete modeling would 

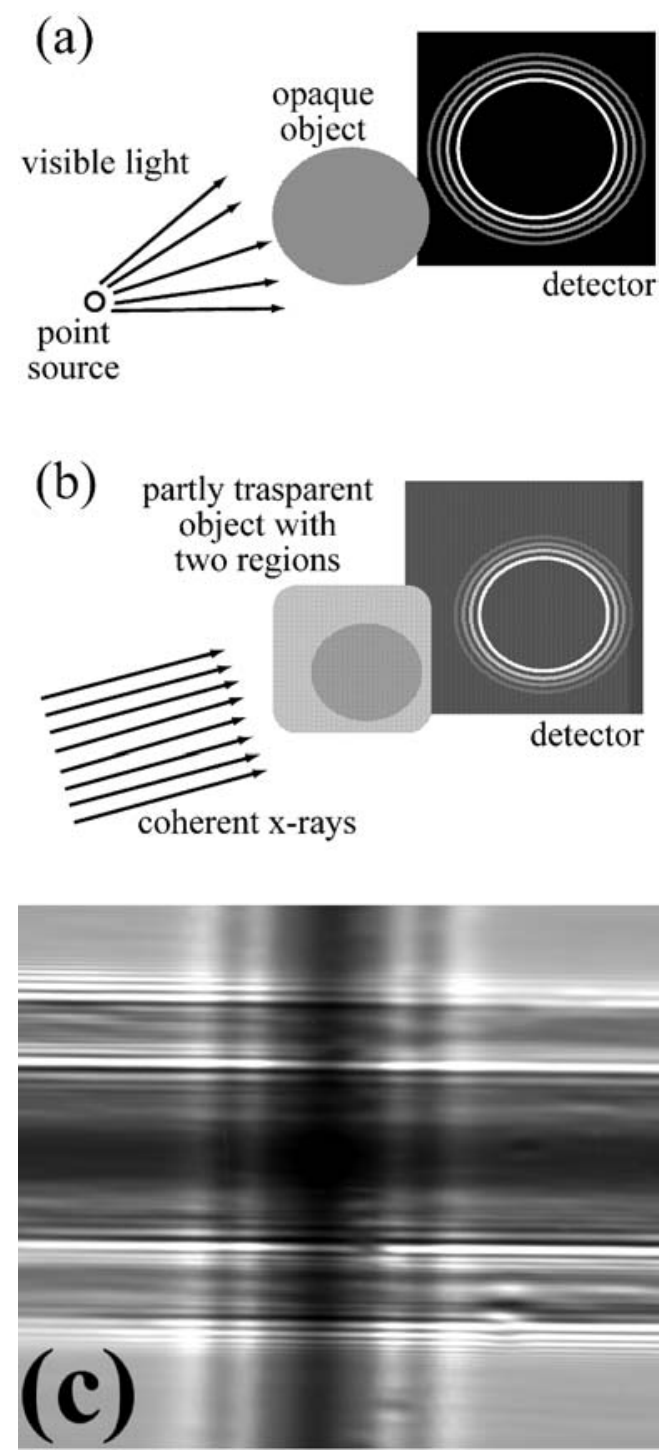

Fig. 8 Edge enhancement by Fresnel edge diffraction. a An opaque object illuminated by visible light of well-defined wavelength emitted by a point-source produces diffraction fringes. b A similar phenomenon occurs when coherent X-rays illuminate a sharp edge between two different regions of an object. c Diffraction fringes are indeed clearly visible in this radiograph of two crossed optical fibers (horizontal and vertical), specifically the fringes are present both for the outer edges between the horizontal fiber and vacuum and for the edges between the inner and outer parts of this fiber. On the contrary, no fringes can be seen for the vertical fiber. The reason is that the synchrotron X-rays beamemitted by a bending magnet-was coherent only in the vertical direction perpendicular to the horizontal fiber edges. This in turn is due to the fact that for a bending magnet source, size and angular spread are small only in the vertical direction

require a detailed treatment of the X-ray propagation, including phenomena such as interference and diffraction.

Without theoretical complications, we can still understand the most important consequences with simple ar- guments [22]. Imagine (Fig. 8a) an opaque object with sharp, non-tapered edges illuminated by a point source of visible light with reasonably well-defined wavelength. It is well known that this illumination produces diffraction fringes at the edge; this is the classical phenomenon known as "Fresnel edge diffraction."

Consider now (Fig. 8b) the equivalent phenomenon for X-rays: a coherent synchrotron X-ray beam illuminating a sharp edge between two object regions. There is, in this case, no "opaque" object since both regions of the system are partly transparent to X-rays. Nevertheless, edge diffraction fringes are produced by the coherent beam-see Fig. 8c. Such diffraction fringes do produce edge enhancement as refraction did for tapered edges.

Note that our discussion of Fig. 8b hypothesized a "sharp edge." Specifically, an infinitely sharp edge can produce diffraction as in Fig. 8b, but it cannot produce edge enhancement by refraction. In a more realistic picture, the object edges are not infinitely sharp, and both refraction and diffraction effects can coexist. We saw that refraction effects tend to decrease at large detectorobject distances: this property can be used to enhance diffraction effects relative to refraction effects or vice versa [21].

Practically speaking, refraction effects dominate the vast majority of edge-enhanced radiographs. In the relatively few cases when they are visible, diffraction effects can have both a positive and a negative impact on the image analysis. On one hand, they complicate the images since each edge corresponds to a series of fringes rather than just to a pair of dark-bright fringes. On the other hand, the edge diffraction fringes potentially carry much more information than the refraction fringes. The diffraction fringes are theoretically equivalent to a hologram and could lead to holographic reconstruction. It is doubtful, however, that this approach could be of interest for medical radiology, whereas it could find applications in other branches of radiology.

\section{Diffraction-enhanced imaging}

We have so far separately treated the contrast related to the refractive index and the conventional absorption contrast. This is not realistic: in practical cases, both mechanisms are active and the overall contrast is due to their interplay. An accurate analysis of the images of Figs. 4, $6,7,8$ reveals indeed that refraction (and/or diffraction) contrast are also accompanied by absorption contrast.

The interplay of absorption and refraction may sometimes complicate the image analysis. Theoretical optics, however, offers a very elegant method to separate the two mechanisms: the "diffraction-enhanced imaging," or DEI [23].

As schematically shown in Fig. 9a, a "Bragg filter" is placed after the specimen and before the detector. The 

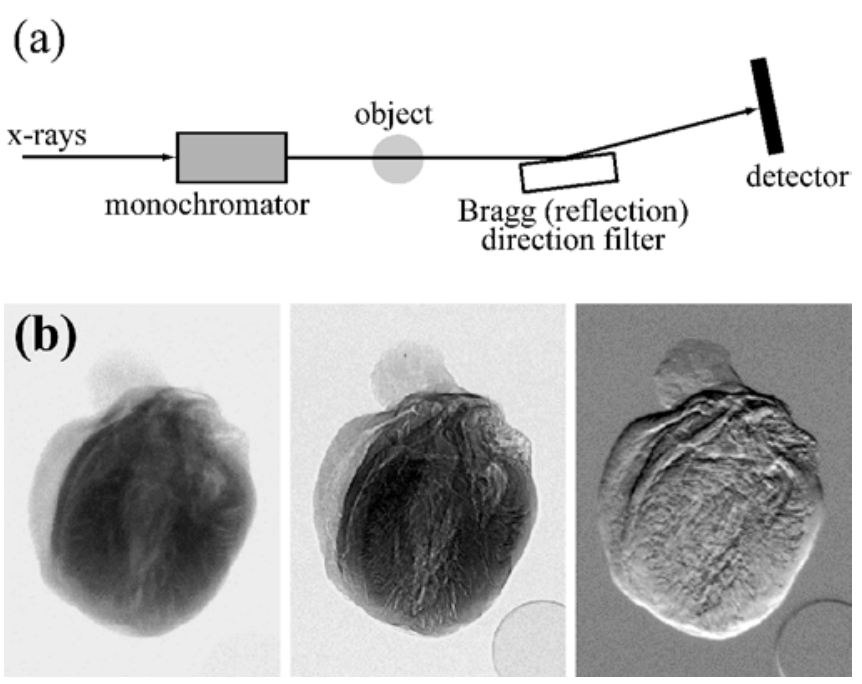

Fig. 9 a Scheme of the diffraction-enhanced imaging (DEI) approach [23]. b Example of DEI result: (left) standard radiograph of a mouse heart; (center and right) processed DEI image corresponding to absorption and refractive-index contrast (see [24], picture courtesy of the British Journal of Radiology)

Bragg filter is a crystal that drastically reduces the intensity of X-rays traveling in all directions except its "Bragg reflection." This sharp direction filtering already improves the radiographs by suppressing the diffused background.

The DEI technique, however, exploits the direction of filtering in far a more sophisticated way. Two images are sequentially taken with the Bragg filter direction slightly tilted at two symmetric angles with respect to the beam direction. A mathematical procedure is then applied to combine the pixels of the two images. This procedure yields two new images, one only due to absorption contrast and the other only to refractive-index contrast [23, 24] - see Fig. 9b.

Other clever ways have been developed to enhance the effects of the different contrast mechanisms [2] - and more undoubtedly will be invented in the future. Such approaches have not yet reached the medical radiology community, except for a few specialists. This is regrettable, since a closer collaboration between synchrotron radiation experts and medical-radiology experts could fine-tune the development of new techniques, as happened in the case of DEI.

\section{Tomography techniques}

An increasingly important role in synchrotron radiology is played by tomography reconstruction [25-27]. Similar to standard medical tomography, this approach consists of taking images for many different object orientations and then using powerful computer-based mathematical meth- ods to reconstruct object slices or other views. The use of synchrotron radiation makes it possible to extend computer-assisted tomography to the micron and submicron scale, with substantial impact on radiological research.

This impact is enhanced by the use of refractionbased contrast. The enhancement because of refraction improves the edge visibility and makes it possible to reduce the X-ray dose: this is important for the multiple image taking required for tomography. The corresponding technique is called "phase contrast microtomography" [25-27].

The tomographic reconstruction is still affected by some difficulties related to the phase-based contrast mechanism. These problems notwithstanding, synchrotron phase contrast microtomography has already produced very spectacular results, and medical applications can be foreseen for special diagnostic problems.

\section{Outlook}

Part I of our review [1] already discussed in realistic terms the advantages and disadvantages of using synchrotron sources for medical applications and the corresponding outlook. We discussed, in particular, issues concerning the infrastructure, the construction and operating costs and the logistics and the inconvenience of using a centralized facility. We thus limit our discussion here to the novel radiology techniques and to the corresponding instrumentation.

Synchrotron-based radiology is very far from the end of its development: the potential has been only marginally exploited so far [2]. Specialized beamlines fully dedicated to radiology tests accelerate this development.

Particular attention is devoted to the X-ray detectors. Standard films or ionization chambers are suitable for detecting synchrotron-emitted X-rays. However, most of the techniques discussed above are enhanced by the use of digital detectors such as CCD detectors with high lateral resolution, fast readout and suitable computer-based reading systems. For microradiography, specialized approaches have been developed [13] based on photoelectron emission microscopes (PEEMs).

We have seen that wavelength tunability is one of the strongest features of synchrotron sources. Other developing approaches besides those discussed above attempt to make a more extensive use of this feature [30]. We saw that images taken at different wavelengths can reveal the localization of a given chemical element such as iodine. This is possible, in principle, for any element with an $\mathrm{X}$-ray absorption edge in the accessible region of wavelengths. In practice, however, this procedure was confined so far to strong X-ray absorbers like iodine or gadolinium.

Synchrotron X-rays and "phase contrast" could overcome this limitation and make it possible to observe the spatial distribution of other elements. This is based on a 
fundamental optical property: an X-ray absorption edge must correspond to a rapid change of the refractive index with the wavelength [2]. Thus, refraction-enhanced images taken at different wavelengths close to an absorption edge could yield information on the spatial distribution of the corresponding chemical element. Practical tests of this hypothesis are underway with preliminary success [30].

In more general terms, the biggest challenge for synchrotron-based radiology is medical validation after extensive tests on human patients. This validation must demonstrate that new techniques are substantially better than standard radiology in their specific domains of application. The advantages must be strong enough to justify overcoming the technical difficulties of using a synchrotron facility.

So far, extensive medical validation tests have only been performed for synchrotron coronary angiography. The results have been impressive and the advantages of synchrotron-based angiography clearly demonstrated. However, the key issue is not yet resolved: the experts are not unanimous about the adoption of synchrotron angiography as a routine analysis.

Medical validation tests are being developed for other techniques such as "phase contrast" synchrotron mammography. Actual tests on human patients are expected to begin at the Elettra synchrotron in Trieste within the next 12-18 months, the main remaining obstacle being the legal requirements. The results will clarify the basic question: will synchrotron radiology remain a "niche" activity or will it join the mainstream of medical radiology?

A mainstream role would, of course, become easier if the technical difficulties could be simplified-in particular, by developing compact sources suitable for a hospital environment. As mentioned in part I, the Comptonbackscattering sources [31] are most promising. Although generally inferior to storage rings, they would be suitable for virtually all synchrotron radiology techniques - and would be much smaller, cheaper and simpler to operate. This, together with the demonstration of their superiority in specific cases, might be the key factor for the future role of synchrotron radiology.

Acknowledgments We are very grateful to Giuliana Tromba for several comments and practical information on phase contrast, mammography and DEI. Our work in synchrotron radiology is performed at the PLS facility in Korea, at SRRC-Taiwan, at the Argonne APS and at Elettra in Trieste. Our research programs in this domain are supported by the National Science Council of Taiwan, by the SKORE-A Program, by KOSEF, by the Fonds National Suisse de la Recherche Scientifique, by the Ecole Polytechnique Fedérale de Lausanne and by the NICOP Program (N0001499-1-0814) of the US Office of Naval Research.

\section{References}

1. Margaritondo G, Meuli R (2003) Synchrotron radiation in radiology. Novel X-ray sources. Eur Radiol 13:25332541

2. Margaritondo G (2003) Elements of synchrotron radiation for chemistry, biology and medical research. Oxford University Press, New York

3. Margaritondo G (1988) Introduction to synchrotron radiation. Oxford University Press, New York

4. Burattini E, Gambaccini M, Marziani M, Rimondi O, Indovina PL, Pocek M, Simonetti G, Benassi M, Tirelli C, Passariello R (1992) X-ray mammography with synchrotron radiation. Rev Sci Instrum 63:638-640

5. Burattini E, Cossu E, Di Maggio C, Gambaccini M, Indovina PL, Marziani M, Pocek M, Simeoni S, Simonetti G (1995) Mammography with synchrotron radiation. Radiology 195:239-244

6. Johnston RE, Washburn D, Pisano E, Thomlinson WC, Chapman D, Gmur NF, Zhong Z, Sayers D (1995) Mammography phantom studies with synchrotron radiation. Radiology 197:221
7. Arfelli F, Bonvicini V, Bravin A, Cantatore G, Castelli E, Dalla Palma L, Di Michiel M, Fabrizioli M, Longo R, Menk RH, Olivo A, Pani S, Pontoni D, Poropat P, Prest M, Rashevsky A, Ratti M, Rigon L, Tromba G, Vacchi A, Vallazza E (2000) Mammography with synchrotron radiation: phase detection techniques. Radiology 215:286-293

8. Dix WR, Kupper W, Dill T, Hamm CW, Job H, Lohmann M, Reime B, Ventura R (2003) Comparison of intravenous coronary angiography using synchrotron radiation with selective coronary angiography. J Synchrotron Radiat 10:219-227

9. Thomlinson W, Gmür N, Chapman D, Garrett R, Lazarz N, Moulin H, Thompson AC, Zeman HD, Brown GS, Morrison J, Reiser P, Padmanabahn V, Ong L, Green S, Giacomini J, Gordon H, Rubenstein E (1992) First operation of the medical-research facility at the NSLS for coronary angiography. Rev Sci Instrum 63:625-628

10. Kolesnikov KA, Kulipanov GN, Kuzin MV, Mezentsev NA, Nesterov SI, Pindyurin VF, Dragun GN, Rozenberg OA, Zelentsov EL (1995) Preliminaryresults of an animals lymphatic-system study at the angiography station of the Vepp-3 storage-ring. Nucl Instrum Methods A 359:364-369
11. Elleaume H, Fiedler S, Esteve F, Bertrand B, Charvet AM, Berkvens P, Berruyer G, Brochard T, Le Duc G, Nemoz C, Renier M, Suortti P, Thomlinson W, Le Bas JF (2000) First human transvenous coronary angiography at the European Synchrotron Radiation Facility. Phys Med Biol 45:L39-L43

12. Takeda T, Itai Y, Hyodo K, Ando M, Akatsuka T, Uyama C (1998) Medical applications with synchrotron radiation in Japan. J Synchrotron Radiat 5:326-332

13. Hwu Y, Tsai Wen-Li, Groso A, Margaritondo G, Je Jung Ho (2002) Coherence-enhanced synchrotron radiology: simple theory and practical application. J Phys D35:R105-R120

14. Arfelli F, Assante M, Bonvicini V, Bravin A, Cantatore G, Castelli E, Dalla Palma L, Di Michiel M, Longo R, Olivo A, Pani S, Pontoni D, Poropat P, Prest P, Rashevsky A, Tromba G, Vacchi A, Vallazza E, Zanconati F (1998) Low-dose phase contrast X-ray medical imaging. Phys Med Biol 43:2845-2852 
15. Olivo A, Castelli E, Arfelli F, Bravin A, Pani S, Tromba G (1998) Phase contrast and diffraction enhanced imaging with synchrotron radiation: a feasibility study for mammographic applications. Radiology 209:608-608

16. Snigirev A, Snigireva I, Kohn V, Kuznetsov S, Schelokov I (1995) On the possibilities of X-ray phase contrast microimaging by coherent high-energy synchrotron radiation. Rev Sci Instrum 66:5486-5492

17. Pogany A, Gao D, Wilkins SW (1997) $\mathrm{X}$-ray phase-contrast radiography. Rev Sci Instrum 68:2774-2782

18. Wilkins SW, Gureyev TE, Gao D, Pogany A, Stevenson AW (1996) Phase-contrast imaging using polychromatic hard X-rays. Nature 384:335-338

19. Takeda T, Momose A, Wu J, Yu Q, Zeniya T, Thet-Thet-Lwin, Yoneyama A, Itai Y (2002) Vessel imaging by interferometric phase-contrast X-ray technique. Circulation 105:1708-1712
20. Cloetens P, Pateyron-Salomé M, Buffière JY, Peix G, Baruchel J, Peyrin F, Schlenker M (1997) Observation of microstructure and damage in materials by phase sensitive radiography and tomography. J Appl Phys 81:5878-5886

21. Margaritondo G, Tromba G (1999) Coherence-based edge diffraction sharpening of X-ray images: a simple model. J Appl Phys 85:3406-3408

22. Hwu Y, Hsieh HH, Lu MJ, Tsai WL, Lin HM, Goh WC, Lai B, Je JH, Kim CK, Noh DY, Youn HS, Tromba G, Margaritondo G (1999) Coherenceenhanced synchrotron radiology: refraction vs. diffraction mechanisms. J Appl Phys 86:4613-4618

23. Chapman D, Thomlinson W, Johnston RE, Washburn D, Pisano E, Gmur N, Zhong Z, Menk R, Arfelli F, Sayers D (1997) Diffraction-enhanced X-ray imaging. Phys Med Biol 42:2015-2025

24. Lewis RA, Hall CJ, Hufton AP, Evans S, Menk RH, Arfelli F, Rigon L, Tromba G, Dance DR, Ellis IO, Evans A, Jacobs E, Pinder SE, Rogers KD (2003) X-ray refraction effects: application to the imaging of biological tissues. Br J Radiol 76:301-308

25. Spanne P, Raven C, Snigireva I, Snigirev A (1999) In-line holography and phase-contrast microtomography with high energy X-rays. Phys Med Biol 44:741-749
26. Momose A, Takeda T, Itai Y, Hirano K (1996) Phase-contrast X-ray computed tomography for observing biological soft tissues. Nat Med 2:473-475

27. Stampanoni M, Borchert G, Abela R, Ruegsegger P (2002) Bragg magnifier: a detector for submicrometer X-ray computer tomography. J Appl Phys 92:7630-7635

28. Lee K-H, Hwu Y, Je JH, Tsai W-L, Choi E-W, Kim Y-C, Kim H-J, Seong J-K, Yi S-W (2002) Synchrotron radiation imaging of internal structures in live animals. Yonsei Med J 43:25-30

29. Tsai WL, Hsu PC, Hwu Y, Chen CH, Chang LW, Je JH, Lin HM, Groso A, Margaritondo G (2002) Building on bubbles in metal electrodeposition. Nature 417:139-139

30. Groso A, Margaritondo G, Hwu Y, Tsai W-L, Je JH, Lai B (2002) Dispersive coherence-enhanced radiology: experimental test and modeling. Appl Phys Lett 81:3888-3892

31. Carroll FE (2002) Tunable monochromatic X-rays: a new paradigm in medicine. Am J Roentgenol 179:583-590 